\title{
Autism Detection in Children by Combined Use of Gaze Preference and the M-CHAT-R in a Resource-Scarce Setting
}

\author{
Kelly Jensen ${ }^{1,2,13} \cdot$ Sassan Noazin $^{3}\left[\right.$ - Leandra Bitterfeld ${ }^{4,14} \cdot$ Andrea Carcelen $^{3} \cdot$ Natalia I. Vargas-Cuentas $^{5}$. \\ Daniela Hidalgo ${ }^{5,6}$. Alejandra Valenzuela ${ }^{5}$. Avid Roman-Gonzalez ${ }^{5}$. Casey Krebs ${ }^{7}$. Vincent Clement ${ }^{6} \cdot$ Cody Nolan $^{8}$. \\ Franklin Barrientos ${ }^{5}$. Ardi Knobel Mendoza ${ }^{9}$. Paola Noriega-Donis ${ }^{10} \cdot$ Claudia Palacios $^{5}$. Andrea Ramirez ${ }^{5}$. \\ Macarena Vittet ${ }^{5}$. Emil Hafeez ${ }^{3}$. Mariana Torres-Viso ${ }^{11}$. Myriam Velarde ${ }^{12}$ - Lawrence H. Moulton ${ }^{3}$. \\ Michael D. Powers ${ }^{7,11} \cdot$ Robert H. Gilman ${ }^{3} \cdot$ Mirko Zimic $^{5}$. On behalf of the Autism Working Group in Peru
}

Accepted: 7 January 2021 / Published online: 16 February 2021

(c) The Author(s) 2021

\begin{abstract}
Most children with autism spectrum disorder (ASD), in resource-limited settings (RLS), are diagnosed after the age of four. Our work confirmed and extended results of Pierce that eye tracking could discriminate between typically developing (TD) children and those with ASD. We demonstrated the initial $15 \mathrm{~s}$ was at least as discriminating as the entire video. We evaluated the GP-MCHAT-R, which combines the first $15 \mathrm{~s}$ of manually-coded gaze preference (GP) video with M-CHAT-R results on 73 TD children and 28 children with ASD, 36-99 months of age. The GP-MCHAT-R (AUC $=0.89$ (95\%CI: 0.82-0.95)), performed significantly better than the MCHAT-R $(\mathrm{AUC}=0.78(95 \% \mathrm{CI}: 0.71-0.85))$ and gaze preference $(\mathrm{AUC}=0.76$ (95\% CI: 0.64-0.88)) alone. This tool may enable early screening for ASD in RLS.
\end{abstract}

Keywords Autism spectrum disorder $\cdot$ Developmental disorder $\cdot$ Eye tracking $\cdot$ Gaze preference $\cdot$ ASD diagnosis

\section{Abbreviations}

Gp Gaze Preference

ET Eye tracking

ADOS-II Autism Diagnostic Observation

Schedule-II

Kelly Jensen and Sassan Noazin contributed equally to this study and manuscript preparation and are co-first authors.

Sassan Noazin

snoazin1@jhu.edu

1 Tulane University School of Medicine, New Orleans, LA, USA

2 School of Public Health and Tropical Medicine, Tulane University, New Orleans, LA, USA

3 Department of International Health, Johns Hopkins Bloomberg School of Public Health, Baltimore, MD, USA

4 Johns Hopkins University School of Nursing, Baltimore, MD, USA

5 Bioinformatics and Molecular Biology Laboratory, Research and Development Laboratory, Science and Philosophy Faculty, Universidad Peruana Cayetano Heredia, Lima, Peru

6 Psychology Faculty, Universidad Peruana Cayetano Heredia, Lima, Peru
GP-MCHAT-R Gaze preference combined with the MCHAT-R for ASD detection

ASD Autism spectrum disorder

TD Typically developing children

MCHAT-R Modified Checklist for Autism in Toddlers-Revised

ABA Applied Behavior Analysis

ADI-R Autism Diagnostic Interview Revised

$7 \quad$ Yale Child Study Center, Yale University School of Medicine, New Haven, CT, USA

8 Weill Cornell Medical College, New York, NY, USA

9 Rutgers Robert Wood Johnson Medical School, New Brunswick, NJ, USA

10 Johns Hopkins University Whiting School of Engineering, Baltimore, MD, USA

11 CCSN: The Center for Children With Special Needs, Glastonbury, CT, USA

12 IMLA: Instituto Medico Lengua Aprendazaje, Lima, Peru

13 Present Address: Louisiana State University, Baton Rouge, USA

14 Present Address: USA Indian Health Service, Albuquerque, USA 
LOWESS Locally weighted scatterplot smoothing

ROC Receiver-operating curves

AUC Area under the ROC curve

Autism spectrum disorder (ASD) encompasses a group of neurodevelopmental disorders characterized by variable deficits in communication that present in early childhood (American Psychiatric Association 2013; CDC 2016). According to the Centers for Disease Control and Prevention (CDC), one in 59 children in the United States is affected by ASD (Christensen et al. 2016). Although the prevalence of ASD has been increasing on a global scale, estimates of the worldwide prevalence are much lower, 1 in 160 children (Elsabbagh et al. 2012; WHO 2020) for several reasons. Among these are a lack of awareness of the disease both among physicians and the community overall, a lack of mental health infrastructure and practitioners and possible stigma regarding developmental delays, all of which lead to a lack of autism screening and detection ("Autism on the Rise" 2013; Christensen et al. 2016). The American Academy of Pediatrics recommends that children undergo screening for ASD at 18 months and 24 months (C. P. Johnson et al. 2007). Typically, a screening test, such as the Modified Checklist for Autism in Toddlers-Revised (M-CHAT-R) is used and is considered positive if two of the critical questions were positive and /or three or more of the non-critical questions were positive. This is followed by a diagnostic work-up to confirm and determine the disorder severity (CDC 2016; Robins et al. 2014). Diagnostic tests include the Autism Diagnostic Observation Schedule-II (ADOS-II), which requires observation of the child by a trained clinician and the Autism Diagnostic Interview Revised (ADI-R) in which the child's parents are interviewed. These diagnostic tests together with a child's overall developmental profile are used to generate a comprehensive clinical diagnosis of ASD (Le Couteur 2003). While the ADOS-II is often considered the "gold standard" evaluation procedures for the identification of ASD, it must be carried out by trained health professionals and is both time and resource intensive (Paula et al. 2011; Delfos 2011). As a result, its applicability and availability to low-resource settings is limited even as the need exists in such settings for screening tools for ASD.

Numerous studies demonstrate improved intellectual and adaptive behavior in children with early intervention utilizing the broad principles and practices of Applied Behavior Analysis (ABA) (Estes et al. 2015; Helt et al. 2008; Reichow et al. 2012; Warren et al. 2011). However, in the absence of widespread and evidence-based screening and diagnostic evaluation procedures, access to otherwise valuable early intervention opportunities is substantially compromised.
The aim of this study was to investigate the validity of a portable gaze-preference system as a low-cost screening tool for ASD and demonstrate consistency of the screening tool at follow-up study visits. Though previous studies on eye tracking for autism diagnosis exist, the software and protocols are expensive, and require specialized equipment thus limiting their applicability. Our a priori hypothesis was that the concurrent use of our low-cost gaze preference system and the MCHAT-R questionnaire as a joint screening tool is able to predict ASD status. Initial examination of our data led to the following a posteriori hypotheses: (a) the joint screening tool can be used for better specificity, (b) the initial $15 \mathrm{~s}$ of the gaze-preference video is at least as predictive as the entire video and (c) the duration of gaze on the social scene is more predictive than that on the abstract scene.

\section{Methods}

\section{Study Design and Participants}

Children 36-99 months old were recruited from educational centers throughout Lima, Peru. Children with ASD were identified through three private child development centers in Lima, including the Instituto Medico Lenguaje y Aprendazaje, (IMLA), where they were receiving therapy for a previously established diagnosis of ASD. The diagnosis of ASD for these children was consistent with criteria from the Diagnostic and Statistical Manual of Mental Disorders$5^{\text {th }}$ Edition (American Psychiatric Association 2013), based on a combination of clinical evaluation and previous exams including evoked auditory potentials, language and sensorial processing evaluations, and psychological evaluations. Participants were excluded if they had a primary developmental disability diagnosis other than ASD, such as a language disorder, general developmental disorder, or learning disability, in addition to autism. The control group consisted of typically developing (TD) children who were identified through public schools and daycare centers in Lima, Peru. Control-group children were eligible if they had no previous diagnosis of ASD, specific language disorder, general developmental disorder, learning disability, intellectual disability, or neurologic illness, and no illness at the time of enrollment.

The MCHAT-R was selected for use as a component of the screening protocol because of its broad availability in a number of languages including Spanish and its ease and accessibility of administration (Canal-Bedia et al. 2011; M-CHATTM_MCHAT R/F Translations 2020). While the MCHAT-R is widely used as a screener for possible ASD (Coury et al. 2017) it is not without its limitations. In addition, traditionally the MCHAT-R is considered most valid as a screening tool for children through 48 months 
of age. However, given that children in Peru do not typically present for, and are not usually identified with, ASD until 36 months or later (Observatorio Nacional de la Discapacidad I CONADIS Peru-OBSERVATORIO DE LA DISCAPACIDAD 2020), we elected to expand the study group age range to 99 months in order to access a larger pool of individuals for this exploratory study.

All children were screened with the MCHAT-R- and ADOS-II at the time of recruitment.

Control-group children who screened positive with the ADOS-II evaluation at the time of enrollment were excluded from analysis, as they lacked a clinical diagnosis of ASD.

\section{Gaze Preference Recording}

We used a standard laptop with a 17-inch screen with two videos, one of social scenes and the other of abstract scenes playing, separated by 8 inches (Vargas-Cuentas et al. 2017). The original video used for the testing, was saved with a resolution of $1920 \times 1080$ pixels, at an aspect ratio of 16:9, and displaying at 60 frames per second. The social and the abstract images displayed were projected in rectangular areas on the left and on the right of the screen. The size of the image was $37.5 \%$ of the width and $37.04 \%$ of the height of the screen. The original video was recorded at a temporal resolution of 60 frames per second and this is the way it is "projected" in the screen. However, the camera that recorded the video of the face of the child, captured a video with a temporal resolution of 30 frames per second.

For the gaze preference screening, each child watched a minute-long video on a computer with a front-mounted camera that recorded their face movements. The video consisted of a 10-s introductory scene to attract the child's attention, followed by a split screen showing the abstract scene on the right side of the screen and the social scene shown on the left. The social scene consisted of a video of young children playing and interacting with each other, and the abstract scene consisted of moving shapes of various colors. These visits took place at the respective recruitment sites. Each child was seated in a chair $30 \mathrm{~cm}$ from the computer screen. If the child was unable to sit alone, they were placed in their guardian's lap $30 \mathrm{~cm}$ from the computer screen. This distance allowed for clear visibility of the eyes by coders without being uncomfortable for the child. If the child's attention turned away from the video, they were redirected either with auditory or visual cues to the screen by their guardian or supervising study personnel. The procedure was repeated approximately one month after the initial recording. No adverse effects were observed associated with this procedure.

\section{Gaze Preference Coding}

The gaze-preference device was based on the study by Vargas-Cuentas et al. 2017. The current study was done to provide the proof-of-concept for the validity of our approach and correctness of our hypotheses. We used manual coding to bypass any coding software issues. We are in the process of improving our coding software to enable fully automated screening process. Study personnel reviewed each video at a rate of 2 frames per second, one for every 15 frames filmed (101 frames per child). At each time point the child's gaze was labeled as either left, right, center or distracted. Left indicated focus on the social scene, right indicated focus on the abstract scene. Distraction was defined as any time that the child was not looking within the frame of the video or their eyes were not visible. Center was when the child was looking at the center of the screen between the social and abstract video. Two separate, independent groups of trained coders who were blinded to the diagnostic status of the subjects coded the videos taken at visit one and visit two. In cases of discrepancy, the two coders reanalyzed the frame in question and decided the gaze direction with consensus. Given the constraints of the study conditions, a more traditional method of determining interrater reliability was not undertaken. Gaze preference was determined by calculating the proportion of the stimulus display time-or equivalently, the proportion of all frames - the child spent looking at the social scene, abstract scene, center of the screen, or not looking at the screen (distraction time).

\section{Statistical Analysis}

Stata 14.2, StataCorp, College Station, Texas, USA was used for statistical analysis. To demonstrate the gaze-preference patterns among ASD and typically developing (TD) children over the course of the video, we used LOWESS smoothing (locally weighted scatterplot smoothing) (Cleveland and Devlin 1988) with a bandwidth of 0.8. A Mann-Whitney Test was used to compare proportions of social and abstract scenes and distraction times between TD children and children with ASD. We verified the consistency of these results between two separate gaze-preference visits by intra-class correlation ICC) between visits. We employed Wilcoxon signed-rank test to compare gaze preference of the same group of children at different segments of the video. To develop a two-step approach to classifying ASD and control children, we estimated a logistic regression model and used the Hosmer-Lemeshow test with group sizes of 5 and 10 in separate tests to examine the model goodness of fit. We also report the chi-sq test of goodness of fit between the predicted and actual ASD status and have included a plot of their predicted and actual values and a plot of predicted probability vs the number of social scenes in the supplement. 
This model used the results of the MCHAT-R in addition to gaze preference as predictors of ASD status. We used AUC to compare models based on the first $15 \mathrm{~s}$ of gaze-preference data against one based on the entire $50 \mathrm{~s}$ length of the video and subsequently we tested the model in one half of the sample and used each to calculate the AUC for the other half of the sample. Additionally, we tested the coefficient of the interaction term between the dummy variable indicating each half of the sample and each of the main model predictors (MCHAT-R and gaze preference results) to test whether the models from the two halves of the sample were different. We used a similar approach to test whether a model based on the age groups shared by cases and controls could predict the ASD status of children outside those age ranges. We also computed sensitivity of the model at specificity levels of approximately $70 \%, 80 \%$ and $90 \%$ to indicate how model sensitivity would change at different levels of specificity. To study the association between children's ADOS-II scores and their gaze preference, the Mann-Whitney test was used. Participating children were excluded from the analysis if they were controls with a positive ADOS-II score $(n=7)$ in which case their true ASD status could not be ascertained or if their MCHAT-R or ADOS-II scores were missing $(n=16)$.
Additionally, there were two controls and one case excluded due to acute illness.

\section{Results}

\section{Focus Area Discrimination Between ASD and TD Groups}

We administered the gaze-preference test at 2 separate occasions, on average 1 month apart. The analyzable sample size in visits one and two were 101 (73 TD controls and 28 children with ASD) and 100 (73 controls and 27 children with ASD), respectively. Cases were predominantly male (89\%) and older (median $=60$ months) compared to controls who were $51 \%$ male and younger (median $=42$ months) (Table 1 ). Please also see tables S.1 in the Supplement.

Gaze preference classification detected differences between the group of children with ASD and the TD control group (Table 2 depicts these differences in visit 1 while visit 2 results are presented in table S. 3 in the Supplement). As seen in Table 2, over the full $50 \mathrm{~s}$, children with ASD spent significantly less time than the TD control
Table 1 Age, sex and ADOS-II score distribution in TD and ASD diagnosed children

\begin{tabular}{|c|c|c|c|c|c|c|c|c|c|}
\hline & \multirow[t]{2}{*}{$\mathrm{N}$} & \multicolumn{4}{|l|}{ Age } & \multicolumn{4}{|c|}{ ADOS-II } \\
\hline & & mean & $\mathrm{sd}$ & $\min$ & $\max$ & mean & $\mathrm{sd}$ & $\min$ & $\max$ \\
\hline \multicolumn{10}{|c|}{ TD } \\
\hline $\mathrm{F}$ & $37(51 \%)$ & 42.6 & 4.5 & 36 & 51 & 1.6 & 2 & 0 & 7 \\
\hline M & $36(49 \%)$ & 43.0 & 4.7 & 36 & 50 & 0.5 & 1.4 & 0 & 7 \\
\hline \multicolumn{10}{|c|}{ ASD } \\
\hline $\mathrm{F}$ & $3(11 \%)$ & 48.0 & 12 & 36 & 60 & 22.7 & 4.9 & 17 & 26 \\
\hline M & $25(89 \%)$ & 61.3 & 14.5 & 36 & 99 & 18.6 & 5.8 & 6 & 26 \\
\hline
\end{tabular}

\begin{tabular}{lllll}
\hline Time course & Focus area & ASD mean (SD) & TD mean (SD) & Significance \\
\hline First 15 s (30 frames) & Social & $0.301(0.215)$ & $0.501(0.196)$ & $\mathrm{P}<0.001$ \\
& Abstract & $0.503(0.238)$ & $0.335(0.176)$ & $\mathrm{P}=0.001$ \\
& Center & $0.010(0.027)$ & $0.035(0.045)$ & $\mathrm{P}=0.002$ \\
& Distracted & $0.185(0.217)$ & $0.129(0.149)$ & $\mathrm{P}=0.175$ \\
First 30 s (60 frames) & Social & $0.237(0.165)$ & $0.398(0.174)$ & $\mathrm{P}<0.001$ \\
& Abstract & $0.542(0.237)$ & $0.419(0.172)$ & $\mathrm{P}=0.013$ \\
& Center & $0.011(0.025)$ & $0.026(0.037)$ & $\mathrm{P}=0.003$ \\
& Distracted & $0.210(0.200)$ & $0.157(0.133)$ & $\mathrm{P}=0.421$ \\
& Social & $0.215(0.163)$ & $0.335(0.154)$ & $\mathrm{P}<0.001$ \\
& Abstract & $0.505(0.227)$ & $0.462(0.167)$ & $\mathrm{P}=0.358$ \\
& Center & $0.013(0.019)$ & $0.021(0.019)$ & $\mathrm{P}=0.049$ \\
& Distracted & $0.267(0.218)$ & $0.183(0.129)$ & $\mathrm{P}=0.170$ \\
\hline
\end{tabular}

Viewing the social scene, the abstract scene, the center of the screen or being distracted during the first 15, first 30 or the entire $50 \mathrm{~s}$ of the video in visit 1 . Example: Children with ASD and TD children, respectively, gazed at the social scene $30.1 \%$ and $50.1 \%$ of all frames during the first $15 \mathrm{~s}$ of the video
Table 2 Gaze-preference behavior, visit 1: table entries indicate the proportion (SD) of all frames gazed at. (Visit 2 is presented in the Supplement) 
group focused on the social scene at both visit 1 (21.5\% vs $33.5 \%$ of the length of the video, $\mathrm{p}<0.0001$ ) and visit $2(24 \%$ vs. $36 \%$, respectively, p $<0.001)$. Furthermore, although children with ASD were not significantly more distracted than TD children in the first visit $(26.7 \%$ vs $18.3 \%, p=0.170)$ (Table 2; Fig. 1a, b) this difference was significant in the second visit (25.0\% vs $12.2 \%, \mathrm{p}=0.013$ ). By contrast, attention to the abstract scene during the $50 \mathrm{~s}$, although higher, was not significantly so, among ASDdiagnosed children in visit $1(50.5 \%$ vs $46.2 \%, \mathrm{p}=0.358)$ or visit $2(50.8 \%$ vs $50.9 \%, p=0.920)$. Gazing at the center of the screen was rare and it was inconsistent between the two visits among TD children. (Table 2 and Fig. $1 \mathrm{a}$ and $\mathrm{b}$, for more detailed graphs and LOWESS with different bandwidth see Supplement Figures S.4-S.6).

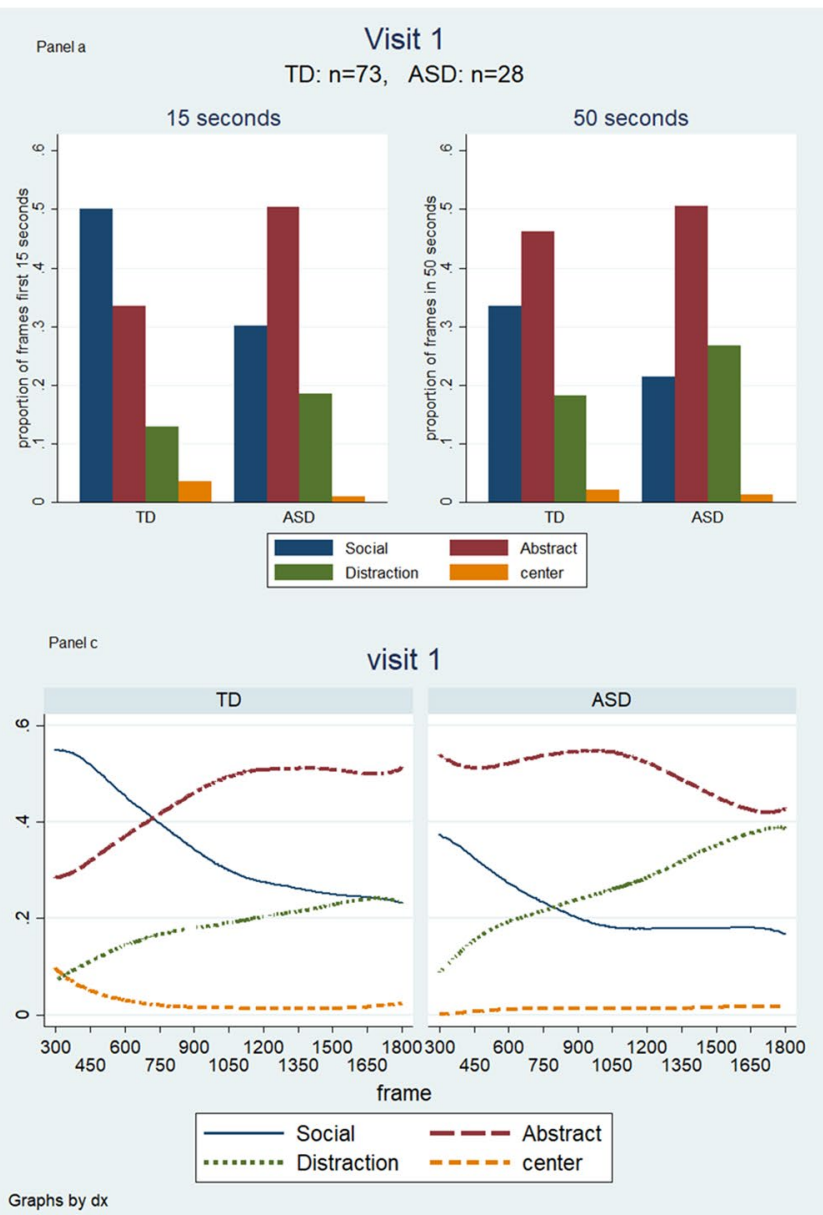

Fig. $1 \mathbf{a}$ and $\mathbf{b}$ : Focus areas of TD children and children with ASD during the first $15 \mathrm{~s}$ and the full $50 \mathrm{~s}$ at visit $1 \mathbf{a} \&$ visit $2 \mathbf{b}$. Each cluster of bars totals $100 \%$. c and $\mathbf{d}$ : Focus trends among the two groups in the $50 \mathrm{~s}$ video in visit $1 \mathbf{c} \&$ visit $2 \mathbf{d}$. In $\mathbf{c}$ and $\mathbf{d}$, LOWESS

\section{Time Course Analysis}

Graphs for visit 1 and visit 2 (Fig. 1a-d) suggest a very high degree of consistency in gaze patterns between the two visits (based on the full length of the video, the between visit ICC for social scene $=0.54,95 \%$ CI: $0.40-0.68$, for abstract scene $=0.44,95 \%$ CI: $0.29-0.60$, for distraction $=0.41$, 95\% CI: $0.24-0.57$, for center $=0,95 \%$ CI: $0-0.20$ ).

Visual inspection of graphs of smoothed trajectories of gaze preference behavior (Fig. 1c and d) suggests that the first $15 \mathrm{~s}$ maximally discriminate between children with ASD and controls in both visits: as most TD children started the video by gazing at the social scene an increasingly fewer proportion of them did so until the trajectory for social scene viewing crossed that for abstract scene at around the $600^{\text {th }}$

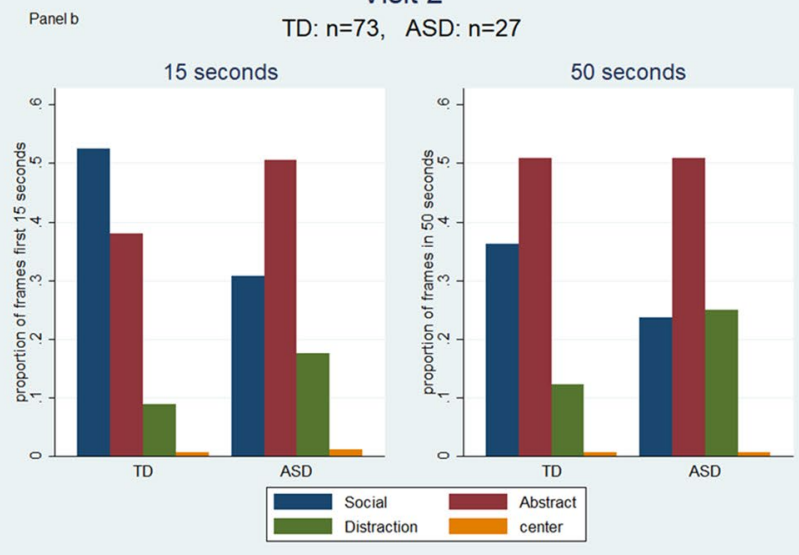

Paneld

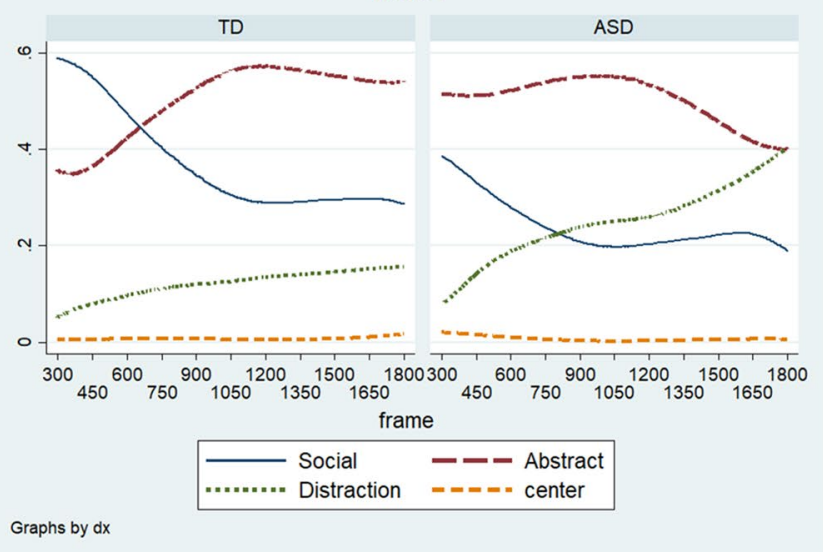

lines demonstrate the smoothed proportion of gaze at each scene by cases and controls at every $1 / 2$ second of the video, depicting change in gaze direction as time elapsed over the duration of the video 
frame $\left(17^{\text {th }}\right.$ second $)$. By contrast, children with ASD tended to view the abstract scene throughout this segment and paid increasingly less attention to the social scene. Based on these observations, we hypothesized that gaze patterns during the first $15 \mathrm{~s}$ of the video discriminated between children with ASD and TD controls as well as gaze patterns over the entire length (50 s) of the video (Fig. 1a and b and Table 2).

Attention to the social scene diminished among both TD children and those with ASD as time elapsed (Wilcoxon signed rank test of difference between 15 and 50 s: $50.1 \%$ vs $33.5 \%, \mathrm{p}<0.001$ for control group, $30.1 \%$ vs $21.5 \%$, $\mathrm{p}<0.001$ for ASD group) while distraction increased in both groups (Wilcoxon signed rank test of difference between 15 and 50 s: $12.9 \%$ vs $18.3 \%, p<0.001$ for control group, $18.5 \%$ vs $26.7 \%, \mathrm{p}<0.041$ for ASD group). Children with ASD spent about $50 \%$ of their time viewing the abstract scene, and this tendency did not change significantly throughout the video (Wilcoxon signed rank test of difference between 15 and $50 \mathrm{~s}: 50.3 \%$ vs $50.5 \%, \mathrm{p}=0.793$ ). By contrast, typically developing children paid significantly less attention to the abstract scene during the first $15 \mathrm{~s}$ of the video but paid more attention as time elapsed (Wilcoxon signed rank test: $33.5 \%$ vs $46.2 \%, \mathrm{p}<0.001)$. These patterns and the significant differences reported in Table 2 suggest that the data from the first $15 \mathrm{~s}$ of the video are as discriminating between the ASD and control groups as the data from the entire length of the video for social focus and distraction, while abstract scene viewing is a significant discriminator only in the first $15 \mathrm{~s}$.

\section{Association of the First $15 \mathrm{~s}$ of Gaze-Preference Behavior with ADOS-II}

Figure 2, corresponding with the first and the second visit, demonstrates that ADOS-II classification was inversely associated with the proportion of social scene frames during the first $15 \mathrm{~s}$; i.e., ADOS-II-positive children viewed the social frame less frequently (Fig. 2). By contrast, ADOS-II classification was directly associated with the proportion of abstract scene frames suggesting that ADOS-II positive children preferred the abstract scene. We also observed that distraction during the first $15 \mathrm{~s}$ of the video was higher in ADOS-II-positive children. These associations were statistically significant.

\section{The Two-Step Approach to Modeling Autism Using Gaze Preference and the MCHAT-R: Development of the GP-MCHAT-R Model}

Gaze preference over the full length of the video could discriminate between TD children and those with ASD, but we also observed that the initial $15 \mathrm{~s}$ of the video was at least as discriminating which prompted us to hypothesize that a shorter video could represent gaze preference before distraction sets in. This was also suggested by Fig. 1, by the steadily rising distraction lines. We hypothesized that the first few seconds before the lines for abstract and social scene cross and when the difference between the proportion of abstract and social gazes are almost opposite (on average) between cases and controls (prior to frame 600) and while distraction is at its lowest, we could get the most discriminating information. A predictive logistic model with one or more gaze-preference variables also suggested this (Table 3ai). In our sample, MCHAT-R with critical questions demonstrated a sensitivity of $93 \%$ (95\% CI: 76-99\%) and a specificity of $63 \%$ (95\% CI: 51-74\%), with an AUC of 0.78 . We used a 2 -step screening including gaze preference and MCHAT-R to improve on the MCHAT-R's low specificity while incorporating its high sensitivity. Of the gazepreference variables, social scene focus was the most predictive option, and we used it in combination with MCHAT-R results to construct a parsimonious, unweighted logistic regression model, the GP-MCHAT-R model (Table 3ai, ii). Adding other gaze preference variables within the same model did not improve the fit or model prediction. We also examined a model with both abstract scene and distraction - but without the social scene - which could predict ASD similarly well, but we used the model with social scene since it was more parsimonious. The Hosmer-Lemeshow test for the model based on social scene indicated good fit throughout the response range ( $p=0.397$ based on quintiles of data and $p=0.577$ based on deciles). In the Supplement, we have presented the details of the Hosmer-Lemeshow test as well as two graphs presenting a histogram of model predicted probabilities in the two groups of children and the predicted probabilities plotted against social15 values (tables S.4 and S.5 and graphs S.1 and S.2). In another step for model validation, we initially estimated the model in each of two random halves of the sample (seed $=99, \mathrm{n} 1=55$ and $\mathrm{n} 2=46$ ) and produced the ROC curve for the estimation half (AUC1 $=0.89$ and $\mathrm{AUC} 2=0.87)$ as well as the validation half $(\mathrm{AUC} 3=0.88$ and AUC4 $=0.89$ ). To ensure the model applicability to different random subsamples of the data, we conducted an analysis to test the coefficient of the interaction terms between the dummy variable representing the two random subsamples separately with social 15 and MCHAT-R in the model. The p-values for these interactions ( 0.681 and 0.622 , respectively) suggest no difference in these coefficients when estimated in each subsample. Results are presented in the Supplement (Table S.6 and Figure S.3). Table 3a and Fig. 3a demonstrate the 15-s model and the 50-s model. To verify this, we conducted a likelihood-ratio test to examine the effect of the presence or absence of each of social15 and social50 in a model with MCHAT-R. The full model consisted of MCHAT-R, social15 and social50 as predictors. We tested this against a reduced model with social 15 but without social50 and one with social50 but 


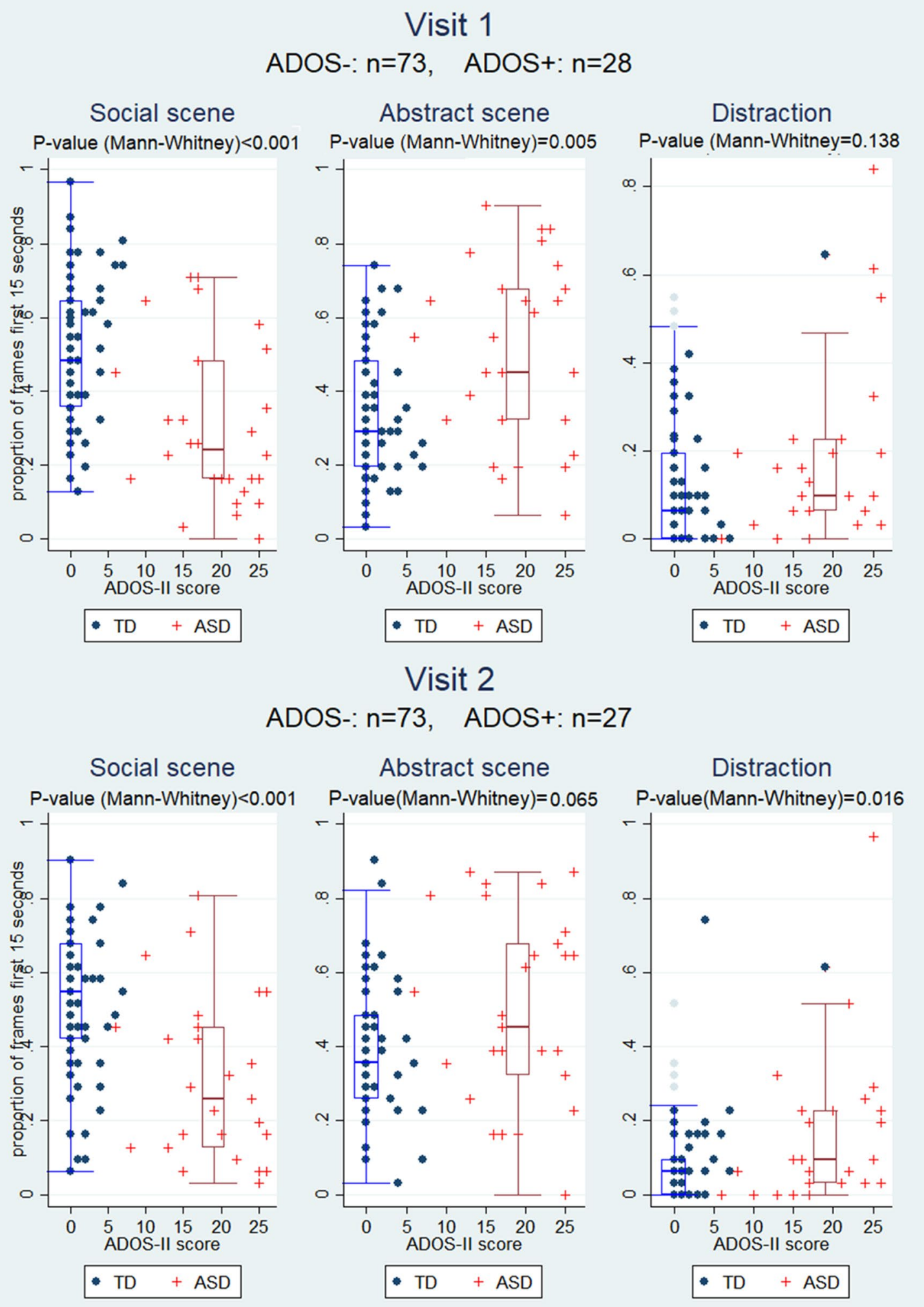

Fig. 2 Association of gaze-preference behavior during the first $15 \mathrm{~s}$ with established ADOS-II diagnosis 


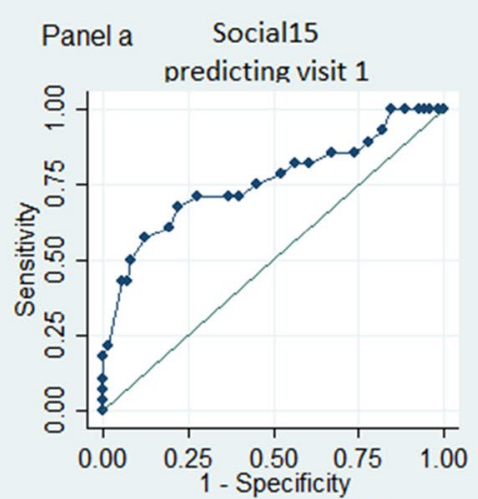

Area under ROC curve $=0.7605$

Social50

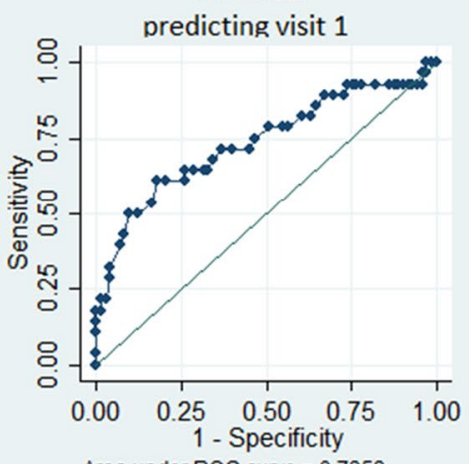

Area under ROC curve $=0.7353$

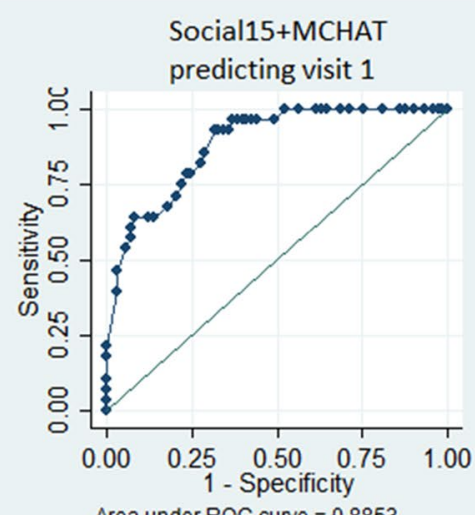

Area under ROC curve $=0.8853$

Social50+MCHAT

predicting visit 1

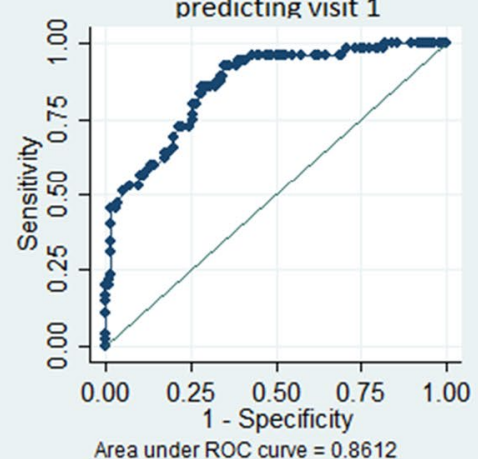

Area under ROC curve $=0.8612$

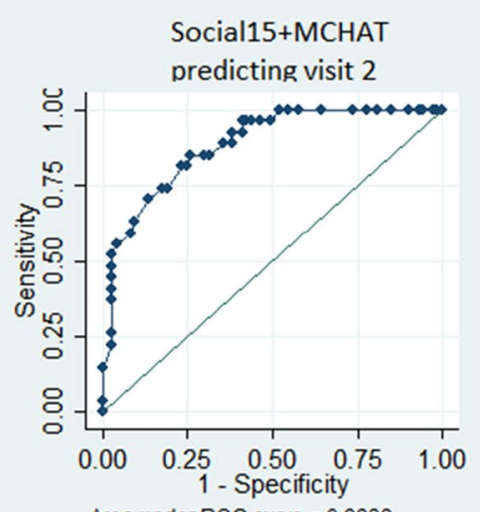

Area under $R O C$ curve $=0.8838$

Social50+MCHAT predicting visit 2

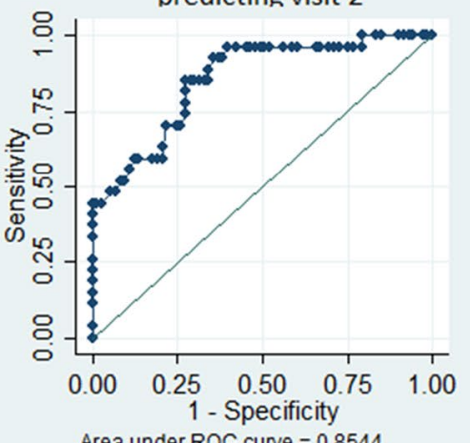

Area under ROC curve $=0.8544$

Panel b

Visit 1


Visit 2
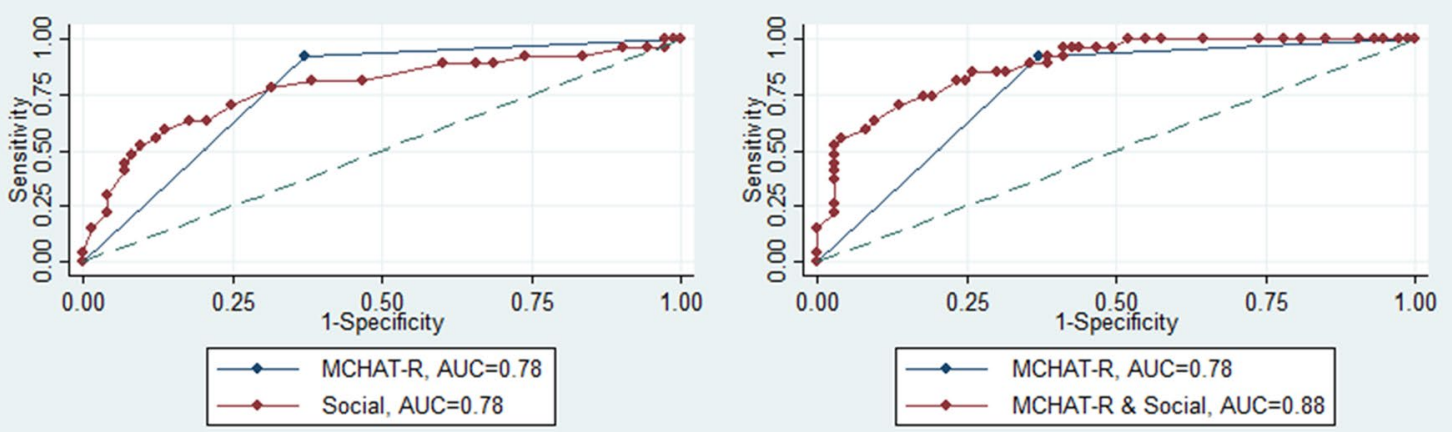
4Fig. 3 a ROC curves and AUC's based on logistic models, first $15 \mathrm{~s}$ and the full $50 \mathrm{~s}$. Models were developed using visit 1 data and were used to classify visit 1 and visit 2 data. Model predictors: Social15 represents the amount of time spent viewing the social scene during the first $15 \mathrm{~s}$ of the gaze preference data. Social50 represents the amount of time spent viewing the social scene during $50 \mathrm{~s}$ of the gaze-preference data. Social15+MCHAT represents the amount of time spent viewing the social scene during the first $15 \mathrm{~s}$ and the MCHAT combined. b predictability of ASD based on attention to the social scene in the first $15 \mathrm{~s}$ alone, MCHAT-R alone or the GPMCHAT-R model, compares the ROC curves and AUC values for the models based on the first $15 \mathrm{~s}$. The left panels compare MCHAT-R against social scene (first $15 \mathrm{~s}$ ) prediction. The right-side panels compare MCHAT-R against the 2-step model prediction

without social15 (likelihood-ratio test comparing each reduced model against the full model: $\mathrm{p}=0.464$ and 0.009 , respectively) and concluded that the absence of social15 in the model leads to a significantly worse model whereas absence of social50 is not a significant detriment.

Because the age distribution of the children with ASD and TD controls were different (see Tables S.1 and S.2 in the Supplement) we fitted the model with social15 and MCHAT-R but included a dummy variable identifying ages that were common to both children with ASD and TD children (45-49 months). We then fitted a logistic model with social15, MCHAT-R, the dummy variable and the interaction terms, similar to what was described above for the random subsample analysis (this is also discussed in the Supplement: Comparing the GP-MCHAT-R Model in the Age Groups Including Both Cases and Controls Against Other Ages). Results suggested that the coefficients for social 15 and MCHAT-R were applicable to both age groups ( $\mathrm{p}$-value for social15 $=0.781$, $\mathrm{p}$-value for MCHAT-R $=0.332$ ).

Figure $3 \mathrm{a}$ depicts the ROC curves and associated AUC's for both models in visit 1 and visit 2 data (AUC values are 0.89 and 0.86 for the 15 - and the 50 -s models). As Fig. $3 \mathrm{~b}$ indicates, although the AUC for gaze preference (social scene focus in the first $15 \mathrm{~s}$ ) is slightly less than that for MCHAT-R alone (0.76 vs $0.78, \mathrm{p}=0.796$ ), the combined AUC for the 2-step model, which includes both MCHAT-R and social scene as predictors, presents a significant improvement over MCHAT-R alone ( 0.89 vs 0.78 , $\mathrm{p}<0.001$ ), particularly on model specificity.

Considering the importance of both sensitivity and specificity for an effective screening tool and the tendency of MCHAT-R to give false alarms, we also assessed the GPMCHAT-R model's sensitivity and specificity in addition to its AUC. Table 3 bi demonstrates the sensitivity of the GPMCHAT-R model associated with specificities of approximately $90 \%, 80 \%$ or $70 \%$ in each of visit 1 and visit 2 . The GP-MCHAT-R model AUC in visit 1 is 0.89 and in visit 2 it is 0.88 (Fig. 3a). This model has a sensitivity of $64 \%$ at a specificity level of exactly $92 \%$ when applied to visit 1 data, and a sensitivity of $63 \%$ given a specificity of $90 \%$ when applied to visit 2 data. Thus, the model can provide an alternative to the low specificity of MCHAT-R when a lower sensitivity is acceptable.

\section{Discussion}

This study demonstrates that combining gaze preference, as psychophysical behavioral coding of preferential looking in infants and toddlers, using data from a 15 -s initial observation period and the MCHAT-R provides better sensitivity and specificity than either test alone. Recently, eye-tracking studies in ASD have included examining visual saccades, speed and accuracy of eye movements, and differences in eye fixation regions on human faces (Chawarska et al. 2016; Harrop et al. 2018; B. P. Johnson et al. 2016; Jones and Klin 2013; Klin et al. 2009; Kovarski et al. 2019; Mottron et al. 2007; Pierce et al. 2011, 2016; Vargas-Cuentas et al. 2017). Overall, our application is consistent with findings from prior gaze preference studies.

Our principal finding was that gaze preference could be a basis for community surveillance of ASD and also that MCHAT-R and gaze-preference results could be combined in a predictive model.

Our work confirmed the results previously described by Pierce et al. (Pierce et al. 2011, 2016) that gaze preference over the full length of the video could discriminate between TD children and those with ASD, but we also observed in our data that the initial $15 \mathrm{~s}$ of the video was somewhat more discriminating. Therefore, we hypothesized that a shorter video could represent gaze preference before distraction sets in. This was also suggested by the LOWESS graphs, by the steadily rising distraction lines. Our hypothesis based on this observation was that the first few seconds before the lines for abstract and social scene cross and when the difference between the proportion of abstract and social gazes are almost opposite (on average) between cases and controls (prior to frame 600, the 17th second of the video) and while distraction is at its lowest, we could get the most discriminating information.

Within the first $15 \mathrm{~s}$ of the videos, we observed that TD children spent significantly more time focusing on the social scene than the abstract scene while for the children with ASD the reverse was true. Later in the video, TD children's attention shifted from the social scene to the abstract scene while those with ASD paid somewhat less attention to the abstract scene and were increasingly distracted but did not shift to the social scene. A shorter video has the advantage of reducing children's fatigue and loss of interest and increased ease of coding. Therefore, we developed our index screening tool, GP-MCHAT-R using gaze preference during the first $15 \mathrm{~s}$ of the video augmented with the MCHAT-R 


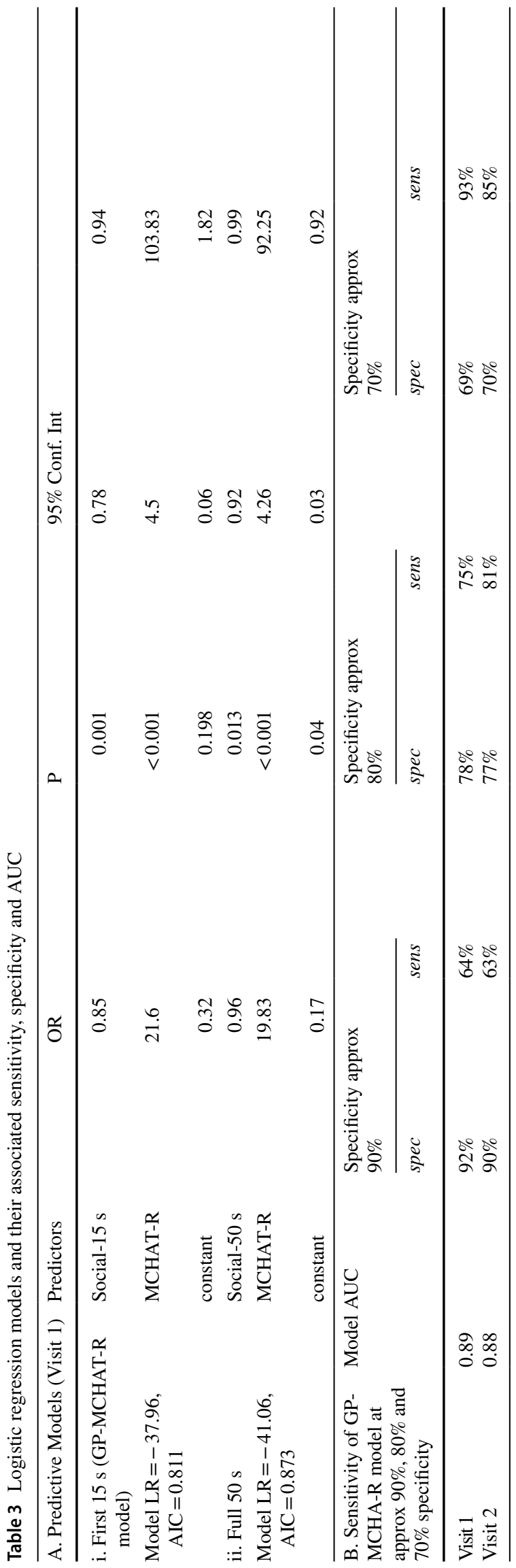

classification results, to predict the risk of ASD with more specificity than either test alone.

Considering the importance of both sensitivity and specificity for an effective screening tool, we chose a model with increased specificity to identify children who may be at increased risk of ASD while minimizing false positives in order to improve on the poor specificity of the MCHAT$\mathrm{R}$. The GP-MCHAT-R model can be adjusted to focus on either specificity or sensitivity, depending on the need of the model. In low-prevalence, resource limited settings, it can be applied with a highly specific cutoff with a sensitivity of $70 \%$ to decrease the number of false positives but with an increase in false negatives. In low-resource settings the increase in specificity decreases the burden on the already limited number of expert clinicians diagnosing ASD.

While there has been an increased focus on gaze-preference differences in ASD, to our knowledge no study has developed a simple, portable gaze-preference screening device. Previous studies have been conducted in a research laboratory, using special equipment and high-power computer software (Jones and Klin 2013; Klin et al. 2009; Kovarski et al. 2019; Mottron et al. 2007). These factors may pose a significant barrier in countries like Peru, which has a widely dispersed rural population and an urban population with difficulties accessing transportation outside of their neighborhood. Thus, families may be unaware of the challenges their child is exhibiting, unaware of screening resources, or may confront stigma associated with ASD. These issues argue strongly for the importance and availability of portable, locally-sourced and delivered screening tools that can be reliably administered by community-based healthcare workers and providers. The application described in this study can be used on any computer with a built-in or mountable camera and data can be collected in as little as $15 \mathrm{~s}$ by a trained community healthcare worker.

There are a number of limitations to this study, many due to setting and access variables noted earlier. Recognizing that in Peru children do not typically present for evaluation of ASD earlier in toddlerhood, we extended the accepted MCHAT-R age range of study participants beyond the typical 48-month cutoff in order to access sufficient children. We deemed this appropriate in order to validate the combined GP-MCHAT-R screening protocol and to investigate "proof-of-concept". Other limitations include the lack of age matching and differences in baseline demographics. Cases were predominantly male and on average over one year older than controls. This was largely unavoidable, considering that autism is more prevalent in males and access to autism screening for toddlers in Peru is limited. Nonetheless, it led to a very small number of girls in the ASD group. Other limitations of the study were the unknown treatment history of children with ASD prior to our study and time from clinical diagnosis of children 
with ASD to the first gaze preference test. Additionally, we did not evaluate the cognitive abilities of the participants and did not collect socio-economic information other than sex and age. As mentioned earlier in Gaze Preference Coding, given the constraints of the study conditions, a more traditional method of determining interrater reliability was not undertaken. This is an important limitation of the study, although we attempted to address this by having the two trained coders resolve any discrepancies in coding child behavior while reviewing the tapes. While not an ideal method, we determined that financial and setting constraints of the study necessitated this less strenuous approach. It is equally important to note that this work is preliminary in nature, was designed to investigate the viability of a simple and straightforward screening system that could be used in resource-scarce areas, in the service of establishing parameters for future investigations of the screening tool that we developed. Finally, due to study limitations we did not include a second control group consisting of children with developmental delays who did not meet diagnostic criteria for ASD. In order to be maximally useful, a screening measure must demonstrate adequate specificity in order to discriminate between children with ASD from those with other developmental challenges. There are several important reasons for this, including in the present case ensuring that scarce human and financial resources are allocated to the children who need them the most. Recognizing that the M-CHAT-R alone may overidentify children with other problems as being at higher risk for ASD, we combined a more robust and well-validated tool-the preferential selection of nonsocial over social stimuli by children with ASD—as an adjunctive strategy for screening, effectively integrating the two tools into a single screening concept. While this does not minimize the importance of comparing and validating the method described in this paper with a follow-up sample of children with ASD, children with an established neurodevelopmental disorder without ASD, and a typically developing sample, we also highlight the exploratory nature of the present work, seeking as it did to determine the efficacy of combining gaze preference with the M-CHAT-R. Our ongoing investigations will address and further refine valid concerns regarding the specificity of the proposed screening tool.

Each of these limitations should be considered and addressed in future efforts to evaluate the viability of the GP-MCHAT-R screener.

The combined GP-MCHAT-R model was designed as an enhanced screening tool and should not be considered a replacement for clinical diagnosis. Rather, a positive screen with the GP-MCHAT-R should guide healthcare professionals to a referral for a more comprehensive ASD diagnostic workup.
In conclusion, we demonstrate that visual preferenceboth social focus and distraction time- - serve as markers for increased risk of ASD in toddlers. Differences are detectable within as little as fifteen seconds of gaze-preference data and combined with the MCHAT-R (GP-MCHAT-R) produces a high degree of reliability with improved sensitivity and specificity, compared with the MCHAT-R alone. Use of the GP-MCHAT-R model has the potential to decrease barriers to early ASD screening in resource-limited settings, ultimately enhancing the potential of better outcomes for toddlers with ASD.

Supplementary Information The online version contains supplementary material available at https://doi.org/10.1007/s10803-021-04878-0.

Acknowledgment This work was supported by INNOVATE Peru, Contrato N 153 PNICP-PIAP-2015, and the GEMS Grants through Johns Hopkins University. Additional funding came from the Wendy Klag grant (160502174, PI: Robert H Gilman) through Johns Hopkins University. Mirko Zimik was a grantee of Bill and Melinda Gates Foundation (OPPOPP1140557). Kelly Jensen was also supported by the Fulbright-Fogarty Fellowship in Peru. The Autism Working Group in Peru Collaborators - Non-author Contributors Vanessa Cavallera, BS; Ricardo Zavaleta, BS; Juan Flores, BS; Dennis Nuñez, BS;Alejandro Dioses, PhD; Anna Smith, MPH.

Author Contributions RHG, MZ, KJ, AR-G, AC and NV-C conceived the study and revised the manuscript; $\mathrm{SN}$ reviewed the initial data analysis and redesigned the analytic approach, conducted the statistical analyses and interpretation and critically revised the intellectual content of the manuscript; Ms LB, Mr EH and Mr FB contributed to the analysis, manuscript preparation and intellectual content; Drs $\mathrm{CK}, \mathrm{CN}, \mathrm{KM}, \mathrm{T}-\mathrm{V}$ and MV and Mr VC, Ms AV, Ms CP, Ms AR, Ms DH, N-D and Ms MV participated in clinical activities and contributed to the manuscript content and preparation; Drs MDP and LHM critically reviewed methods and revised the intellectual content of the manuscript. All authors approved the final manuscript as submitted and agree to be accountable for all aspects of the work. SN, MZ and RG had full access to all the data in the study and take responsibility for the integrity of the data and the accuracy of the data analysis. Analysis was conducted mainly by S N. MZ, LB, RG and KJ also contributed to the analysis. We would like to thank AK, RG and BS for their comments, direction and encouragement and Ms IC and Ms ADD for their contribution to manuscript preparation.

\section{Compliance with Ethical Standards}

Conflict of interest Authors declare that they have no conflict of interest or financial relationship relative to disclose.

Ethical Approval This study was approved by the Institutional Review Board at Johns Hopkins School of Public Health and AB PRISMA in Lima, Peru.

Informed Consent All authors certify that the manuscript represents valid work and that neither this manuscript nor one with substantially similar content under their authorship has been published or is being considered for publication elsewhere.

Open Access This article is licensed under a Creative Commons Attribution 4.0 International License, which permits use, sharing, 
adaptation, distribution and reproduction in any medium or format, as long as you give appropriate credit to the original author(s) and the source, provide a link to the Creative Commons licence, and indicate if changes were made. The images or other third party material in this article are included in the article's Creative Commons licence, unless indicated otherwise in a credit line to the material. If material is not included in the article's Creative Commons licence and your intended use is not permitted by statutory regulation or exceeds the permitted use, you will need to obtain permission directly from the copyright holder. To view a copy of this licence, visit http://creativecommons .org/licenses/by/4.0/.

\section{References}

American Psychiatric Association. (2013). Diagnostic and statistical manual of mental disorders: DSM-5. http://openurl.uqueb ec.ca:9003/uqam?url_ver=Z39.88-2004\&url_ctx_fmt=info:ofi/ fmt:kev:mtx:ctx\&ctx_enc=info:ofi/enc:UTF-8\&ctx_ver=Z39.882004\&rfr_id=info:sid/sfxit.com:azlist\&sfx.ignore_date_thres hold $=1 \&$ rft.isbn $=9780890425572$

Autism on the Rise: A Global Perspective. (2013). Harvard College Global Health Review. https://www.hcs.harvard.edu/hghr/online/ autism-on-the-rise-a-global-perspective/

Canal-Bedia, R., García-Primo, P., Martín-Cilleros, M. V., SantosBorbujo, J., Guisuraga-Fernández, Z., Herráez-García, L., et al. (2011). Modified checklist for autism in toddlers: Cross-cultural adaptation and validation in Spain. Journal of Autism and Developmental Disorders, 41(10), 1342-1351. https://doi.org/10.1007/ s10803-010-1163-z.

CDC. (2016, April 18). Diagnostic Criteria | Autism Spectrum Disor$\operatorname{der}(A S D)|N C B D D D| C D C$. Centers for Disease Control and Prevention. https://www.cdc.gov/ncbddd/autism/hcp-dsm.html

Chawarska, K., Macari, S., Powell, K., DiNicola, L., \& Shic, F. (2016). Enhanced Social Attention in Female Infant Siblings at Risk for Autism. Journal of the American Academy of Child \& Adolescent Psychiatry, 55(3), 188-195.e1. https://doi.org/10.1016/j. jaac.2015.11.016.

Christensen, D. L., Baio, J., Braun, K. V. N., Bilder, D., Charles, J., Constantino, J. N., et al. (2016). Prevalence and Characteristics of Autism Spectrum Disorder Among Children Aged 8 YearsAutism and Developmental Disabilities Monitoring Network, 11 Sites, United States, 2012. MMWR. Surveillance Summaries, 65(3), 1-23. https://doi.org/10.15585/mmwr.ss6503a1.

Cleveland, W. S., \& Devlin, S. J. (1988). Locally Weighted Regression: An Approach to Regression Analysis by Local Fitting. Journal of the American Statistical Association, 83(403), 596-610. https:// doi.org/10.1080/01621459.1988.10478639.

Coury, D., Wolfe, A., Lipkin, P., Baer, B., Hyman, S., Levy, S., Macias, M., \& Sisk, B. (2017). Screening of Young Children for Autism Spectrum Disorders: Results from a National Survey of Pediatricians [Poster]. 2017 Pediatric Academic Societies Annual Meeting. https://www.aap.org/en-us/professional-resources/Research/ research-findings/Pages/Screening-of-Young-Children-for-Autis $\mathrm{m}$-Spectrum-Disorders-Results-from-a-National-Survey-of-Ped. aspx

Elsabbagh, M., Divan, G., Koh, Y.-J., Kim, Y. S., Kauchali, S., Marcín, C., et al. (2012). Global Prevalence of Autism and Other Pervasive Developmental Disorders. Autism Research, 5(3), 160-179. https ://doi.org/10.1002/aur.239.

Estes, A., Munson, J., Rogers, S. J., Greenson, J., Winter, J., \& Dawson, G. (2015). Long-Term Outcomes of Early Intervention in 6-YearOld Children With Autism Spectrum Disorder. Journal of the
American Academy of Child and Adolescent Psychiatry, 54(7), 580-587. https://doi.org/10.1016/j.jaac.2015.04.005.

Harrop, C., Jones, D., Zheng, S., Nowell, S. W., Boyd, B. A., \& Sasson, N. (2018). Sex differences in social attention in autism spectrum disorder. Autism Research: Official Journal of the International Society for Autism Research, 11(9), 1264-1275. https://doi. org/10.1002/aur.1997.

Helt, M., Kelley, E., Kinsbourne, M., Pandey, J., Boorstein, H., Herbert, M., \& Fein, D. (2008). Can children with autism recover? If so, how? Neuropsychology Review, 18(4), 339-366. https://doi. org/10.1007/s11065-008-9075-9.

Johnson, B. P., Lum, J. A. G., Rinehart, N. J., \& Fielding, J. (2016). Ocular motor disturbances in autism spectrum disorders: Systematic review and comprehensive meta-analysis. Neuroscience and Biobehavioral Reviews, 69, 260-279. https://doi.org/10.1016/j. neubiorev.2016.08.007.

Johnson, C. P., Myers, S. M., \& the Council on Children With Disabilities. . (2007). Identification and Evaluation of Children With Autism Spectrum Disorders. Pediatrics, 120(5), 1183-1215. https ://doi.org/10.1542/peds.2007-2361.

Jones, W., \& Klin, A. (2013). Attention to eyes is present but in decline in 2-6-month-old infants later diagnosed with autism. Nature, 504(7480), 427-431. https://doi.org/10.1038/nature12715.

Klin, A., Lin, D. J., Gorrindo, P., Ramsay, G., \& Jones, W. (2009). Two-year-olds with autism orient to non-social contingencies rather than biological motion. Nature, 459(7244), 257-261. https ://doi.org/10.1038/nature07868.

Kovarski, K., Siwiaszczyk, M., Malvy, J., Batty, M., \& Latinus, M. (2019). Faster eye movements in children with autism spectrum disorder. Autism Research: Official Journal of the International Society for Autism Research, 12(2), 212-224. https://doi. org/10.1002/aur.2054.

Le Couteur, A. (2003). National Autism Plan for Children (NAPC). National Autistic Society for NIASA, with RCPsych, RCPCH, APPGA.

M-CHAT ${ }^{\mathrm{TM}}$-MCHAT R/F Translations. (n.d.). $\mathrm{M}-\mathrm{CHAT}^{\mathrm{TM}}$. Retrieved July 28, 2020, from https://mchatscreen.com/mchat-rf/ translations/

Mottron, L., Mineau, S., Martel, G., Bernier, C.S.-C., Berthiaume, C., Dawson, M., et al. (2007). Lateral glances toward moving stimuli among young children with autism: Early regulation of locally oriented perception? Development and Psychopathology, 19(1), 23-36. https://doi.org/10.1017/S0954579407070022.

Observatorio Nacional de la Discapacidad I CONADIS Peru - OBSERVATORIO DE LA DISCAPACIDAD. (n.d.). Retrieved July 28, 2020, from https://www.conadisperu.gob.pe/observatorio/estad isticas/inscripciones-en-el-registro-nacional-de-la-persona-condiscapacidad-a-abril-2020/

Paula, C. S., Fombonne, E., Gadia, C., Tuchman, R., \& Rosanoff, M. (2011). Autism in Brazil-Perspectives from science and society. Revista Da Associação Médica Brasileira, 57(1), 2-5. https://doi. org/10.1590/S0104-42302011000100002.

Pierce, K., Conant, D., Hazin, R., Stoner, R., \& Desmond, J. (2011). Preference for geometric patterns early in life as a risk factor for autism. Archives of General Psychiatry, 68(1), 101-109. https:// doi.org/10.1001/archgenpsychiatry.2010.113.

Pierce, K., Marinero, S., Hazin, R., McKenna, B., Barnes, C. C., \& Malige, A. (2016). Eye Tracking Reveals Abnormal Visual Preference for Geometric Images as an Early Biomarker of an Autism Spectrum Disorder Subtype Associated With Increased Symptom Severity. Biological Psychiatry, 79(8), 657-666. https://doi. org/10.1016/j.biopsych.2015.03.032.

Reichow, B., Barton, E. E., Boyd, B. A., \& Hume, K. (2012). Early intensive behavioral intervention (EIBI) for young children with autism spectrum disorders (ASD). Cochrane Database of Systematic Reviews. https://doi.org/10.1002/14651858.CD009260.pub2. 
Robins, D. L., Casagrande, K., Barton, M., Chen, C.-M.A., DumontMathieu, T., \& Fein, D. (2014). Validation of the Modified Checklist for Autism in Toddlers, Revised With Follow-up (M-CHATR/F). Pediatrics, 133(1), 37-45. https://doi.org/10.1542/ peds.2013-1813.

Vargas-Cuentas, N. I., Roman-Gonzalez, A., Gilman, R. H., Barrientos, F., Ting, J., Hidalgo, D., et al. (2017). Developing an eye-tracking algorithm as a potential tool for early diagnosis of autism spectrum disorder in children. PLOS ONE, 12(11), e0188826. https:// doi.org/10.1371/journal.pone.0188826.

Warren, Z., McPheeters, M. L., Sathe, N., Foss-Feig, J. H., Glasser, A., \& Veenstra-Vanderweele, J. (2011). A systematic review of early intensive intervention for autism spectrum disorders. Pediatrics, 127(5), e1303-1311. https://doi.org/10.1542/peds.2011-0426.

WHO. (2020). Autism spectrum disorders. WHO. Retrieved March

22, 2020, from https://www.who.int/news-room/fact-sheets/detai 1/autism-spectrum-disorders

Publisher's Note Springer Nature remains neutral with regard to jurisdictional claims in published maps and institutional affiliations. 